\title{
PHYSIOLOGICAL AND PHYTOCHEMICAL RESPONSES OF BABY SPINACH (SPINACIA OLERACEA L.) CULTIVARS TO COMBINED NPKS NUTRITION AND BACILLUS SUBTILIS BD233 INOCULATION USING LC-MS
}

\author{
TheKA-Kutumela, M. P. ${ }^{1}$ - KanU, S. A. ${ }^{1}$ - ARAYA, H. T. ${ }^{2}-$ Sedibe, M. M. ${ }^{3}-$ Mudau, F. N. ${ }^{1 *}$ \\ ${ }^{1}$ Department of Agriculture and Animal Health, College of Agriculture and Environmental \\ Sciences, University of South Africa, Private Bag X6, Florida 1710, South Africa \\ ${ }^{2}$ Agriculture Research Council, Vegetable and Ornamental Plant Institute, Private Bag X293, \\ Pretoria 0001, South Africa \\ ${ }^{3}$ Department of Agriculture, Central University of Technology, Free State, Private Bag X20539, \\ Bloemfontein 9301, South Africa \\ *Corresponding author \\ e-mail: thekamp@unisa.ac.za \\ (Received $30^{\text {th }}$ Aug 2019; accepted $25^{\text {th }}$ Nov 2019)
}

\begin{abstract}
Baby spinach (Spinacia oleracea L.) is grown for its nutritional benefits, edible leaves and medicinal purpose. The use of crop amendments such as inoculation with plant growth-promoting bacteria (PGPB) together with fertilizers for crop cultivation is more sustainable as it reduces the excessive use of fertilizers and increases crop yield. The aim of this study was to evaluate the physiological and phytochemical response of baby spinach cultivars to different levels of Nitrogen, Phosphorus, Potassium and Sulphur (NPKS) nutrition, amended with Bacillus subtilis strain BD233 inoculation. A factorial field experiment with treatments arranged in a randomized complete block design (RCBD) was carried out. The results showed that NPKS fertilization significantly affected the total biomass of baby spinach but not B. subtilis (BD233) inoculation and cultivar or their interaction. The level of chlorophyll varied between cultivars ranging from $19.96 \pm 1.62^{\mathrm{b}} \mathrm{mg} / \mathrm{m}^{2}$ and $24.79 \pm 1.98^{\mathrm{a}} \mathrm{mg} / \mathrm{m}^{2}$, however, no significant differences occurred in stomatal conductance among the three cultivars. However, significant difference was observed on compounds among cultivars with NPKS fertilizer application in most identified compounds such as patuletin-3-glucosyl-(1-6)[apiosyl(1-2)]-glucoside, spinacetin-3-glucosyl-(1-6)[apiosyl(1-2)]-glucoside, (S)-Malate and N-Acetyl-D-tryptophan. Therefore, the study recommends that fertilizer at $22 \mathrm{~N}: 22 \mathrm{P}: 30 \mathrm{~K}: 5 \mathrm{~S} \mathrm{~kg} / \mathrm{ha}$ and $33 \mathrm{~N}: 33 \mathrm{P}: 45 \mathrm{~K}: 7 \mathrm{~S} \mathrm{~kg} / \mathrm{ha}$ amended with $B$. subtilis inoculation be considered when cultivating baby spinach.

Keywords: chlorophyll content, green leafy vegetables, multivariate analysis, Plant Growth Promoting Bacteria (PGPB), stomatal conductance
\end{abstract}

\section{Introduction}

Baby spinach (Spinacia oleracea L.) is grown for its nutritional benefits, edible leaves and medicinal purpose (Schrader and Mayberry, 2003; Kerr, 2014; Mudau et al., 2015). It is extensively cultivated in Europe due to favorable conditions and high demand (Kerr, 2014). In South Africa, baby spinach is available to consumers as a fresh, salad mix and as a frozen and canned product (Zikalala et al., 2016). Its growing popularity led to baby spinach being grown worldwide. However, essential nutrients and phytochemical concentrations of baby spinach are affected by climatic conditions, agronomic practices and postharvest factors such as stage of harvesting (Kalt, 2005; Bergquist, 2006), cultivars (Masufi et al., 2019), storage temperature and time (Bergquist, 2006). 
The production of plants requires an accurate supply of nutrients whilst a slight variation in nutrient balance may have a negative effect on crop growth and nutrition status (Sedibe and Allemann, 2012). The availability of nitrogen, phosphorus and potassium in soils during production are essential to improve growth and vegetable quality (King et al., 2008). However, sustainable and proper use of synthetic chemical fertilizer is essential. Using improved production practices and technologies enhances crop growth (Shine and Guruprasad, 2012) and these techniques mitigate the effect of poor growing conditions including nutrient imbalance (Sedibe, 2012). The use of crop amendments together with fertilizers is more sustainable as it reduces excessive use of fertilizer (Diacono and Montemurro, 2010). These amendments respond differently from that of fertilizers, in as much as it only influences plant vigour (Yakhin et al., 2016).

The use of plant growth promoting bacteria (PGPB) in crop amendments is gaining popularity (Diacono and Montemurro, 2010). Inoculation with Bacillus strains showed increase in vegetative growth of crops (Pupathy and Radziah, 2015; Çakmakçi et al., 2007), however, their effect on chemical composition has not been well documented. Bacillus strains are among the most commonly used phosphate solubilizers and have been reported to increase phosphorus uptake (García-López and Delgado, 2016) there by improving plant growth and is less toxic to human and widely exists in soils (Wu et al., 2016). The aim of this study was to evaluate the effect of combined Nitrogen, Phosphorus, Potassium and Sulphur (NPKS) fertilization at different levels on physiological and phytochemical composition of baby spinach cultivars amended with Bacillus subtilis BD233.

\section{Materials and Methods}

\section{Experimental site}

The study was conducted at Agricultural Research Council - Vegetable and Ornamental Plant Institute (ARC-VOPI) in Roodeplaat farm, situated in the sourish mix of bushveld, $25 \mathrm{~km}$ north of central Pretoria, KwaMhlanga (R573) road; GPS coordinates: $25,56 \mathrm{~S} ; 28,35 \mathrm{E}$ (Gauteng province, South Africa). The area is a relatively cool subtropical climate with summer rainfall and cold, dry winter.

\section{Bacterial strain and preparation}

Bacillus subtilis strain BD233 was obtained from the Agricultural Research CouncilPlant Protection Research Institute (ARC-PPRI) in Pretoria, South Africa. Bacillus subtilis strain BD233 were cultured using a LB agar plates after incubation under dark conditions at $28^{\circ} \mathrm{C}$ for $24 \mathrm{~h}$. The bacterial cells were harvested from LB agar plates into liquid LB media to yield 8.547x109 colony forming units (cfu) $\mathrm{mL}^{-1}$ determined by serial dilution with plate counts (Zhang et al., 2008). Baby spinach cv. Anna, Edna and Ohio seeds were surface decontaminated by washing in $0.35 \%$ (v/v) sodium hypochlorite and stirred for $5 \mathrm{~min}$. The used sodium hypochlorite solution was discarded, and decontaminated seeds were washed three times with distilled water. Seeds were then left to dry under the laminar flow prior to planting in seedling trays filled with compost growth medium on 9 January 2016. Germination took place 7 days after planting and seedlings were transplanted after 3 weeks when the plants had 4 leaves each. 


\section{Experimental design and layout}

The experiment was a $5 \times 2$ factorial arranged in a randomized complete block design (RCBD) with three replicates. Five NPKS fertilizer ratios consisted of 0 (0:0:0:0 kg/ha), $25 \%(11: 11: 15: 2 \mathrm{~kg} / \mathrm{ha}), 50 \%(22: 22: 30: 5 \mathrm{~kg} / \mathrm{ha}), 75 \%(33: 33: 45: 7 \mathrm{~kg} / \mathrm{ha})$ and 100\% $(45: 45: 60: 10 \mathrm{~kg} / \mathrm{ha})$ of the recommended fertilizer application for baby spinach (Nemadodzi et al., 2017) and Bacillus subtilis strain BD233 amendments (zero B. subtilis (B-) and B. subtilis (B+) application) to three cultivars of baby spinach (Anna, Edna and Ohio) were evaluated.

Experimental plot size $\left(2.2 \times 2.2 \mathrm{~m}^{2}\right)$ used consisted of in-row and interrow spacing of $20 \mathrm{~cm}$ and $10 \mathrm{~cm}$, respectively. Fertilizers were applied a week after transplanting followed by inoculation with $100 \mathrm{ml}$ of Bacillus subtilis strain BD233 LB per plant a week after fertilizer application. Lime ammonium nitrate $(28 \% \mathrm{~N} \mathrm{~kg} / \mathrm{ha})$ was applied as the $\mathrm{N}$ fertiliser source, phosphorus was supplied in the form of superphosphate $(83 \%$ $\mathrm{P} \mathrm{kg} / \mathrm{ha})$, potassium was supplied in the form of potassium chloride $(50 \% \mathrm{~K} \mathrm{~kg} / \mathrm{ha})$ and sulphur was applied in the form of gypsum $(17 \% \mathrm{~S} \mathrm{~kg} / \mathrm{ha})$. Irrigation was based on the soil moisture conditions for a period of $2.5 \mathrm{~h}$ per irrigation using sprinkler irrigation and weeds were removed manually by hands.

\section{Physiological parameters}

Plants were harvested at 35 days after planting and washed with running water. Chlorophyll content, upper-leaf stomatal and lower-leaf stomatal conductance and total biomass (fresh mass and dry mass) were measured on three baby spinach cultivars namely, Anna, Edna and Ohio. Chlorophyll content was measured using Spad 502 Chlorophyll Meter (Minolta Camera Co. Ltd., Japan) a non-destructive method on healthy mature leaves with homogeneous green colour. Stomatal conductance was measured between 11:00 to 13:00 using SC-1 Leaf Porometer instrument (Decagon Devices USA). Leaf porometer determine stomatal conductance using the actual vapour flux from the leaf through the stomata. At harvesting, freshly harvested material of baby spinach was oven dried at $45^{\circ} \mathrm{C}$ for $24 \mathrm{~h}$ (Bashan et al., 2017). Both fress mass and dry mass were weight to determine the total biomass.

\section{Ultra High Performance Liquid Chromatography-Mass Spectrometer (UHPLC-MS) solvent extraction and preparation}

Mature leaves of baby spinach were harvested, and oven dried at $45^{\circ} \mathrm{C}$ for $24 \mathrm{~h}$ before grounded into a fine powder using a pestle and mortar and stored in airtight tubes in an $80^{\circ} \mathrm{C}$ refrigerator. Thereafter, about $50 \mathrm{mg}$ of ground leaves were weighed subsequent extraction using $15 \mathrm{ml}$ methanol-water. The mixture was sonicated for $20 \mathrm{~min}$ at room temperature and centrifuged at $1300 \mathrm{rpm}$ for $15 \mathrm{~min}$. The mixture was filtered through $0.45 \mu \mathrm{m}$ syringe filters and the supernatant $(1 \mathrm{ml})$ was transferred into $2 \mathrm{ml}$ amber glass vials for UHPLC-MS analysis (Mncwangi et al., 2014).

\section{UHPLC-MS analysis}

The method described by Mncwangi et al. (2014) was adopted with minor changes. The UHPLC analysis was performed on a Waters Acquity Ultra-High-Performance Liquid Chromatography system with PDA detector (Waters, Milford, MA, USA). UHPLC separation was achieved on a UHPLC Ultra C18 column $(100 \mathrm{~mm} \times 2.1 \mathrm{~mm}$, i.d., $5-\mu \mathrm{m}$ particle size, Restek) maintained at $35^{\circ} \mathrm{C}$. The mobile phase consisted of $0.1 \%$ 
formic acid in water (solvent A) and LC-MS grade methanol (solvent B) at a flow rate of $0.3 \mathrm{ml} / \mathrm{min}$. The gradient elution was applied as follows: $85 \% \mathrm{~A}: 15 \% \mathrm{~B}$ to $65 \% \mathrm{~A}: 35 \%$ $\mathrm{B}$ in $4 \mathrm{~min}$, thereafter, changed to $50 \%: 50 \%$ in two min, to $20 \% \mathrm{~A}: 80 \% \mathrm{~B}$ in $1 \mathrm{~min}$, maintaining for $1 \mathrm{~min}$ and back to an initial ratio in $0.5 \mathrm{~min}$. The analysis time was $9 \mathrm{~min}$. Samples were introduced into the mobile phase with an injection volume of $1.0 \mu \mathrm{l}$ (full-loop injection) for samples and $2.0 \mu \mathrm{l}$ for reference standards. The UHPLC system was interfaced with a Xevo G2QTof MS (Waters, USA). The following mass spectrometry operating conditions were applied: source - ESI negative mode; capillary voltage $-3 \mathrm{~kW}$; cone voltage $30 \mathrm{~V}$; calibration - sodium formate; lock spray - leucine enkaphalin and scan mass range - at $200-1500 \mathrm{~m} / \mathrm{z}$.

\section{Statistical analysis}

Analysis of variance (ANOVA) data of agronomic parameters was conducted using Statistica version 10.0 and all-pairwise comparison tests were performed to detect differences among means at a significance level of $\mathrm{p} \leq 0.05$. Significant means were separated using the Duncan Multiple Range Test. UHPLC-MS data was processed by XCMS version 3.5.1 (2016) and analysis of variance across metabolites treatment means was performed using SAS statistical package version 20. The chemometric analysis was performed using the MetaboAnalyst 3.0. (2017). Partial Least Squares - Discriminant Analysis (PLS-DA) was performed to identify compounds responsible for differentiation among cultivars and treatments. Compounds were identified using the Compass data analysis 4.3 and annotated by MetFrag version 2.1. (2010).

\section{Results and Discussion}

\section{Physiological parameters}

This study evaluated the effect of different levels of NPKS fertilization, on some physiological parameters (chlorophyll content, stomatal conductance and total biomass) of three baby spinach cultivars amended with $B$. subtilis. The results showed significant varietal differences in baby spinach's response to the treatments. Among the three cultivars, cv, Edna had the highest chlorophyll content $\left(24 \pm 1.84 \mathrm{mg} / \mathrm{m}^{2}\right)$ and stomatal conductance $\left(27.24 \pm 1.84\right.$ and $10.03 \pm 0.96 \mathrm{~m}^{2} / \mathrm{smol}^{-1}$ upper and lower, respectively) compared to $\mathrm{cv}$. Ohio with the lowest values (Table 1). The differences in baby spinach could be attributed to the genotypic predisposition of the cultivars tested in this study. The findings of the present study are consistent with the findings of Makus (2013) who reported differences in chlorophyll content of spinach cultivars, Samish and Lazio, grown under the same soil type treated with sulphur. Singh et al. (2014) also reported differences in chlorophyll content among cultivars, Siberian kale and Japanese kale.

There was a significant difference observed in the upper and lower stomatal conductance among the three cultivars, with cultivar Edna having the highest upper stomatal conductance and lower stomatal conductance (Table 1). The findings of the current study concured with the findings of Khan et al. (2009), who reported a high significant decrease in stomatal conductance of mustard cv. SS2 than Pusa Jai Kisan with increasing $\mathrm{NaCl}$ concentration. The results also indicated that cultivar type had no effect on the total biomass of baby spinach, ranging from $0.31 \mathrm{~g}$ to $0.40 \mathrm{~g}$. The results correlated with the findings by Masufi et al. (2019) who reported no significant difference on baby spinach cultivar ohio, guitar F1, Lazio F1, monstrous, viroflay and dash. 
Table 1. Effect of NPKS nutrition and Bacillus subtilis strain BD233 inoculation on the physiology of three baby spinach cultivars

\begin{tabular}{|c|c|c|c|c|}
\hline Treatment & $\begin{array}{c}\text { Total biomass /plant } \\
\text { (g) }\end{array}$ & $\begin{array}{c}\text { Chlorophyll } \\
\text { content } \\
\left(\mathbf{m g} / \mathbf{m}^{2}\right) \\
\end{array}$ & \begin{tabular}{|c|}
$\begin{array}{c}\text { Stomatal } \\
\text { conductance (Upper) } \\
\left(\mathbf{m}^{2} / \mathrm{smol}^{-1}\right)\end{array}$ \\
\end{tabular} & $\begin{array}{c}\begin{array}{c}\text { Stomatal } \\
\text { conductance } \\
(\text { Lower })\left(\mathrm{m}^{2} / \mathrm{smol}^{-1}\right)\end{array} \\
\end{array}$ \\
\hline $\begin{array}{c}\text { Fertilization (NPKS) } \\
0 \%(0: 0: 0: 0) \\
25 \%(11: 11: 15: 2 \mathrm{~kg} / \mathrm{ha}) \\
50 \%(22: 22: 30: 5 \mathrm{~kg} / \mathrm{ha}) \\
75 \%(33: 33: 45: 7 \mathrm{~kg} / \mathrm{ha}) \\
100 \%(45: 45: 60: 10 \mathrm{~kg} / \mathrm{ha})\end{array}$ & $\begin{array}{l}0.10 \pm 0.01^{\mathrm{c}} \\
0.17 \pm 0.01^{\mathrm{c}} \\
0.29 \pm 0.04^{\mathrm{b}} \\
0.54 \pm 0.06^{\mathrm{a}} \\
0.60 \pm 0.07^{\mathrm{a}}\end{array}$ & $\begin{array}{c}7.85 \pm 1.17^{\mathrm{e}} \\
18.67 \pm 1.49^{\mathrm{d}} \\
26.55 \pm 1.94^{\mathrm{c}} \\
31.14 \pm 1.81^{\mathrm{b}} \\
35.77 \pm 1.56^{\mathrm{a}}\end{array}$ & $\begin{array}{l}15.80 \pm 1.32^{\mathrm{a}} \\
9.07 \pm 1.33^{\mathrm{b}} \\
5.52 \pm 0.75^{\mathrm{c}} \\
4.53 \pm 0.64^{\mathrm{c}} \\
3.32 \pm 0.51^{\mathrm{c}}\end{array}$ & $\begin{array}{l}17.74 \pm 1.07^{\mathrm{a}} \\
7.93 \pm 0.62^{\mathrm{b}} \\
4.92 \pm 0.61^{\mathrm{bc}} \\
6.20 \pm 0.93^{\mathrm{bc}} \\
6.84 \pm 00.97^{\mathrm{c}}\end{array}$ \\
\hline $\begin{array}{c}\text { B. subtilis (B) inoculation } \\
\text { B- } \\
\text { B }+ \\
\end{array}$ & $\begin{array}{l}0.32 \pm 0.03^{\mathrm{a}} \\
0.36 \pm 0.04^{\mathrm{a}}\end{array}$ & $\begin{array}{l}23.91 \pm 1.55^{\mathrm{a}} \\
24.08 \pm 1.49^{\mathrm{a}}\end{array}$ & $\begin{array}{l}7.22 \pm 0.71^{\mathrm{a}} \\
8.08 \pm 0.89^{\mathrm{a}}\end{array}$ & $\begin{array}{l}8.18 \pm 0.72^{\mathrm{a}} \\
9.27 \pm 0.86^{\mathrm{a}}\end{array}$ \\
\hline $\begin{array}{c}\text { Cultivar }(\mathbf{C}) \\
\text { Anna } \\
\text { Edna } \\
\text { Ohio }\end{array}$ & $\begin{array}{l}0.31 \pm 0.04^{\mathrm{a}} \\
0.32 \pm 0.04^{\mathrm{a}} \\
0.40 \pm 0.06^{\mathrm{a}}\end{array}$ & $\begin{array}{l}24.79 \pm 1.98^{\mathrm{a}} \\
27.24 \pm 1.84^{\mathrm{a}} \\
19.96 \pm 1.62^{\mathrm{b}}\end{array}$ & $\begin{array}{l}7.13 \pm 0.92^{\mathrm{b}} \\
8.83 \pm 0.80^{\mathrm{a}} \\
6.99 \pm 0.91^{\mathrm{b}}\end{array}$ & $\begin{array}{c}8.38 \pm 0.88^{\mathrm{b}} \\
10.03 \pm 0.96^{\mathrm{a}} \\
7.77 \pm 0.74^{\mathrm{b}}\end{array}$ \\
\hline $\begin{array}{c}\text { Cultivar x B } \\
\text { Anna x B- } \\
\text { Anna x B+ } \\
\text { Edna x B- } \\
\text { Edna x B+ } \\
\text { Ohio x B- } \\
\text { Ohio x B+ }\end{array}$ & $\begin{array}{l}0.28 \pm 0.06^{\mathrm{a}} \\
0.33 \pm 0.05^{\mathrm{a}} \\
0.35 \pm 0.06^{\mathrm{a}} \\
0.28 \pm 0.06^{\mathrm{a}} \\
0.44 \pm 0.09^{\mathrm{a}} \\
0.36 \pm 0.07^{\mathrm{a}}\end{array}$ & $\begin{array}{c}24.66 \pm 3.02^{\mathrm{ab}} \\
24.92 \pm 2.63^{\mathrm{ab}} \\
26.06 \pm 2.58^{\mathrm{ab}} \\
28.43 \pm 2.66^{\mathrm{a}} \\
21.04 \pm 2.42^{\mathrm{bc}} \\
18.89 \pm 2.16^{\mathrm{c}}\end{array}$ & $\begin{array}{l}7.60 \pm 1.45^{\mathrm{ab}} \\
6.66 \pm 1.16^{\mathrm{ab}} \\
9.04 \pm 1.16^{\mathrm{a}} \\
8.62 \pm 1.75^{\mathrm{a}} \\
5.01 \pm 0.89^{\mathrm{b}} \\
8.97 \pm 1.66^{\mathrm{a}}\end{array}$ & $\begin{array}{l}7.79 \pm 1.17^{\mathrm{b}} \\
8.96 \pm 1.35^{\mathrm{ab}} \\
9.64 \pm 1.18^{\mathrm{ab}} \\
10.42 \pm 1.55^{\mathrm{a}} \\
7.11 \pm 1.35^{\mathrm{b}} \\
8.43 \pm 1.60^{\mathrm{b}}\end{array}$ \\
\hline $\begin{array}{c}\text { F-Statistics } \\
\text { NPKS } \\
\text { B. subtilis (B) } \\
\text { Cultivar (C) } \\
\text { C x B } \\
\text { NPKS x B } \\
\text { NPKS x B x V }\end{array}$ & $\begin{array}{l}20.64^{* * *} \\
0.70^{\mathrm{ns}} \\
1.88^{\mathrm{ns}} \\
0.90^{\mathrm{ns}} \\
1.32^{\mathrm{ns}} \\
0.48^{\mathrm{ns}}\end{array}$ & $\begin{array}{c}53.32^{* * *} \\
0.02^{\mathrm{ns}} \\
10.04^{* * *} \\
1.93^{*} \\
1.87^{\mathrm{ns}} \\
0.86^{\mathrm{ns}}\end{array}$ & $\begin{array}{c}27.24^{* * *} \\
1.01^{\mathrm{ns}} \\
1.88^{* *} \\
3.25^{*} \\
0.88^{\mathrm{ns}} \\
1.05^{\mathrm{ns}}\end{array}$ & $\begin{array}{c}25.52^{* * * *} \\
1.65^{\text {ns }} \\
2.53^{* *} \\
1.03^{*} \\
0.92^{\text {ns }} \\
9.96^{\text {ns }}\end{array}$ \\
\hline
\end{tabular}

Values $(\mathrm{M} \pm$ S.E.) followed by similar letters in a column are significantly different at $* \mathrm{p} \leq 0.05$, $* * \mathrm{p} \leq 0.001, * * * \mathrm{p} \leq 0.0001$ and ${ }^{\mathrm{ns}}=$ not significant. B- =Zero inoculation with Bacillus subtilis strain $\mathrm{BD} 233, \mathrm{~B}+=$ inoculation with Bacillus subtilis strain BD233

In this study, different levels of NPKS fertilization significantly affected the measured physiological parameters. Baby spinach treated with a higher dose of fertilizer had higher chlorophyll content compared to control and in general, there was a steady increase in chlorophyll content with increase in NPKS fertilization level (Table 1). Similar trend was observed by Pramanik and Bera (2013), who reported gradual increase in total chlorophyll content of hybrid rice with increasing nitrogen levels from zero to $200 \mathrm{~kg} \mathrm{ha}^{-1}$. These results confirm that nitrogen is important in the formation of chlorophyll molecules (Gairola et al., 2009). However, in contrast, the increase in NPKS fertilization caused decreases in the stomatal conductance. The stomatal conductance of the control plant $\left(0.10 \pm 0.01 \mathrm{~m}^{2} / \mathrm{smol}^{-1}\right.$ (upper) and $17.74 \pm 1.07 \mathrm{~m}^{2} / \mathrm{smol}^{-1}$ (lower)) was the highest compared to the other treatments (Table 1). In agreement with results of the study, Nemadodzi et al. (2017), reported similar findings in chlorophyll content and stomatal conductance with the application of NPK fertilization at a ratio of 45:45:60 on baby spinach. The NPKS fertilization level significanly $(\mathrm{p}<0.001)$ affected the total biomass of baby spinach. In general, plant dry biomas increased with increases in the level of fertilization. However, the current study for plants fertilized with the highest NPKS level 45:45:60:10 kg/ha had the highest total biomass $(0.60 \mathrm{~g})$ compared to control. The results are in agreement with the findings of Nemadodzi et al. (2017) who reported that $\mathrm{N}$ and $\mathrm{P}$ fertilization significantly 
affected both the leaf fresh and dry weights in their study. The results contradicted by the findings of Boroujerdnia and Ansari (2007) who reported an increase in dry weight of romaine lettuce with an increase in N levels from $60 \mathrm{~kg} / \mathrm{ha}$ to $180 \mathrm{~kg} / \mathrm{ha}$. Furthermore, Singh et al. (2004) conducted a study on the effect of NPK fertilizers on the growth of basil where it was found that fertilizer application at $75: 40: 40 \mathrm{~kg} / \mathrm{ha}$ significantly increased the dry weight over the control of basil. In okra, no significant difference was reported in dry weight with increasing NPK fertilizers from 10 to 13 grams (Gloria et al. 2017).

From the results obtained in this study, it was evident that Bacillus subtilis (BD233) amendments influenced the chlorophyll content of baby spinach. Bacillus subtilis (BD233) had a significant $(\mathrm{p} \leq 0.05)$ effect on the chlorophyll content of baby spinach, however, there was no significant differences in stomatal conductance (Table 1). These results concurred with findings of Turan et al. (2014) who assessed the effect of B. subtilis on cabbage seedling growth and observed an increase in chlorophyll content compared to the control. Elsewhere, Ekinci et al. (2014) also found similar findings on cauliflower transplants grown under greenhouse conditions. Anjum et al. (2007) reported that an increase in leaf chlorophyll content stimulate plant growth. The stomatal conductance was not affected by the application of Bacillus subtilis BD 233, these findings accorded well with those of Porcel et al. (2014) who reported no effect on stomatal conductance when inoculation with Bacillus strain on tomato plants. The results also showed that Bacillus subtilis (BD233) exhibited no effect on the total biomass of baby spinach cultivars, the results concurred with the findings of Canbolat et al. (2006) who observed none statistical difference between bacterial inoculation and $\mathrm{P}$ fertilizer in terms of dry weight of barley seedling.

The interaction of cultivar and B. subtilis (BD233) was significant for the chlorophyll content and stomatal conductance of baby spinach. In addition, cv. Edna amended with Bacillus subtilis (BD233) had higher chlorophyll content $\left(28.43 \pm 2.66 \mathrm{mg} / \mathrm{m}^{2}\right)$ and stomatal conductance $\left(9.04 \pm 1.75\right.$ and $10.42 \pm 1.55 \mathrm{~m}^{2} / \mathrm{smol}^{-1}$ for upper and lower, respectively) compared to the other cultivars with or without amendment with the bacteria (Table 1).

\section{Phytochemical response}

There has been a lack of a considerable research report on metabolites response of baby spinach to Bacillus subtilis BD 233 and different levels of NPKS using an untargeted approach. Thus, MS/MS was carried out to identify compounds and their differences with respect to the response of three selected baby spinach cultivars to the different treatments employed in this study. The results of this study demonstrated a wide range of compounds which included the patuletin-3-glucosyl-(1-6)[apiosyl(1-2)]-glucoside (m/z 787, Rt. 5.39), spinacetin-3-glucosyl-(1-6)[apiosyl(1-2)]-glucoside (m/z 801, Rt. 5.94), spinacetin-3-(2ффferoylglucosyl)(1-6)[apiosyl(1-2)]-glucoside (m/z 977, Rt. 6.40), and (S)-Malate (m/z 133, Rt. 0.86). Baby spinach metabolites showed significant differences in response to cultivar and NPKS fertilization. Cultivar Ohio exhibited high concentration in most compound which were slightly significant when compared to cv. Anna and Edna (Table 2a,b). However, there were no significant differences in cultivar on compound suvorexant $(\mathrm{m} / \mathrm{z}$ 449, Rt 6.20), 4-(beta-D-Glucopyranosyloxy)-2-hydroxy-6-pentadecylbenzoic acid ( $\mathrm{m} / \mathrm{z}$ 525, Rt 6.84) and (S)-malate (m/z 133, Rt 0.86). Among the compounds identified, $B$. subtilis inoculation and the interaction of NPKS fertilization with B. subtilis only had a slight significant difference $(\mathrm{p} \leq 0.05)$ on compound 2-(1-hydroxyethyl thiamine diphosphate (2-)) (m/z 465, Rt 5.20) (Table 2a,b). Similar compounds were previously reported by Bergquist et al. (2005), who observed a relatively stable total flavonoid content during normal retail storage conditions. 
Table 2a. Concentration mean of compounds identified from three selected baby spinach cultivars with different levels of NPKS nutrition and Bacillus subtilis BD 233inoculation

\begin{tabular}{|c|c|c|c|c|c|c|c|}
\hline \multicolumn{8}{|c|}{ Parameters } \\
\hline & $\mathbf{A}$ & B & $\mathrm{C}$ & D & $\mathbf{E}$ & $\mathbf{F}$ & $\mathbf{G}$ \\
\hline $\operatorname{Mass}(\mathbf{m} / \mathbf{z})$ & 272 & 191 & 465 & 787 & 245 & 801 & 449 \\
\hline RT (min) & 0.61 & 1.06 & 5.20 & 5.39 & 5.55 & 5.94 & 6.20 \\
\hline Treatment & & & & & & & \\
\hline $\begin{array}{c}\text { NPKS } \\
0 \\
25 \\
50 \\
75 \\
100 \\
\end{array}$ & $\begin{array}{c}73501^{\mathrm{a}} \\
78459^{\mathrm{a}} \\
72290^{\mathrm{ab}} \\
62129^{\mathrm{ab}} \\
55789^{\mathrm{b}}\end{array}$ & $\begin{array}{l}12176^{\mathrm{a}} \\
53902^{\mathrm{a}} \\
44023^{\mathrm{a}} \\
38556^{\mathrm{a}} \\
30516^{\mathrm{a}}\end{array}$ & $\begin{array}{l}311216^{\mathrm{b}} \\
567483^{\mathrm{a}} \\
541828^{\mathrm{a}} \\
497287^{\mathrm{a}} \\
478447^{\mathrm{a}}\end{array}$ & $\begin{array}{c}263265^{\mathrm{c}} \\
881780^{\mathrm{a}} \\
679078^{\mathrm{ab}} \\
591637^{\mathrm{b}} \\
514456^{\mathrm{b}}\end{array}$ & $\begin{array}{c}356533^{\mathrm{c}} \\
751495^{\mathrm{a}} \\
809106^{\mathrm{ab}} \\
1012062^{\mathrm{b}} \\
926952^{\mathrm{b}}\end{array}$ & $\begin{array}{c}8414^{\mathrm{c}} \\
14160^{\mathrm{a}} \\
12896^{\mathrm{a}} \\
11930^{\mathrm{ab}} \\
9424^{\mathrm{bc}}\end{array}$ & $\begin{array}{c}82772^{\mathrm{b}} \\
238209^{\mathrm{a}} \\
227119^{\mathrm{a}} \\
275271^{\mathrm{a}} \\
251944^{\mathrm{a}}\end{array}$ \\
\hline $\begin{array}{c}\text { Bacillus }(\mathbf{B}) \\
B- \\
B+ \\
\end{array}$ & $\begin{array}{l}67010^{\mathrm{a}} \\
20570^{\mathrm{a}} \\
\end{array}$ & $\begin{array}{l}38193^{\mathrm{a}} \\
41900^{\mathrm{a}} \\
\end{array}$ & $\begin{array}{l}476703^{\mathrm{a}} \\
542397^{\mathrm{b}} \\
\end{array}$ & $\begin{array}{l}631791^{\mathrm{a}} \\
654665^{\mathrm{a}} \\
\end{array}$ & $\begin{array}{l}834954^{\mathrm{a}} \\
854248^{\mathrm{a}} \\
\end{array}$ & $\begin{array}{l}11693^{\mathrm{a}} \\
12085^{\mathrm{a}} \\
\end{array}$ & $\begin{array}{r}230982^{\mathrm{a}} \\
246132^{\mathrm{a}} \\
\end{array}$ \\
\hline $\begin{array}{c}\text { Cultivar (C) } \\
\text { Anna } \\
\text { Edna } \\
\text { Ohio } \\
\end{array}$ & $\begin{array}{l}58797^{\mathrm{a}} \\
16491^{\mathrm{b}} \\
82351^{\mathrm{b}} \\
\end{array}$ & $\begin{array}{l}20288^{\mathrm{c}} \\
62754^{\mathrm{a}} \\
33700^{\mathrm{b}} \\
\end{array}$ & $\begin{array}{l}461177^{\mathrm{b}} \\
454200^{\mathrm{b}} \\
603385^{\mathrm{a}} \\
\end{array}$ & $\begin{array}{c}307612^{\mathrm{b}} \\
305791^{\mathrm{b}} \\
1259710^{\mathrm{a}} \\
\end{array}$ & $\begin{array}{c}735737^{\mathrm{b}} \\
1062855^{\mathrm{b}} \\
716530^{\mathrm{a}} \\
\end{array}$ & $\begin{array}{c}9669^{\mathrm{b}} \\
2869^{\mathrm{b}} \\
15898^{\mathrm{a}} \\
\end{array}$ & $\begin{array}{l}201460^{\mathrm{a}} \\
273492^{\mathrm{a}} \\
234115^{\mathrm{a}} \\
\end{array}$ \\
\hline $\begin{array}{c}\text { NPKS x } B \\
\text { 0B- } \\
\text { 0B+ } \\
25 \mathrm{~B}- \\
25 \mathrm{~B}+ \\
50 \mathrm{~B}- \\
50 \mathrm{~B}+ \\
75 \mathrm{~B}- \\
75 \mathrm{~B}+ \\
100 \mathrm{~B}- \\
100 \mathrm{~B}+ \\
\end{array}$ & $\begin{array}{c}58678^{\mathrm{a}} \\
103149^{\mathrm{a}} \\
26898^{\mathrm{a}} \\
17801^{\mathrm{a}} \\
17665^{\mathrm{a}} \\
25565^{\mathrm{a}} \\
17234^{\mathrm{a}} \\
15195^{\mathrm{a}} \\
49501^{\mathrm{a}} \\
62077^{\mathrm{a}} \\
\end{array}$ & $\begin{array}{l}10562^{\mathrm{a}} \\
15402^{\mathrm{a}} \\
53140^{\mathrm{a}} \\
54665^{\mathrm{a}} \\
40268^{\mathrm{a}} \\
47779^{\mathrm{a}} \\
40833^{\mathrm{a}} \\
36279^{\mathrm{a}} \\
27739^{\mathrm{a}} \\
33293^{\mathrm{a}} \\
\end{array}$ & $\begin{array}{c}273789^{\mathrm{d}} \\
386071^{\mathrm{bc}} \\
133162^{\mathrm{a}} \\
206216^{\mathrm{ab}} \\
81409^{\mathrm{abc}} \\
86643^{\mathrm{abc}} \\
71578^{\mathrm{abc}} \\
60887^{\mathrm{bc}} \\
157851^{\mathrm{c}} \\
136191^{\mathrm{bc}}\end{array}$ & $\begin{array}{c}101151^{\mathrm{a}} \\
587494^{\mathrm{a}} \\
609444^{\mathrm{a}} \\
620400^{\mathrm{a}} \\
446177^{\mathrm{a}} \\
546529^{\mathrm{a}} \\
494673^{\mathrm{a}} \\
46292^{\mathrm{a}} \\
545039^{\mathrm{a}} \\
555642^{\mathrm{a}} \\
\end{array}$ & $\begin{array}{c}394371^{\mathrm{a}} \\
280857^{\mathrm{a}} \\
219078^{\mathrm{a}} \\
286249^{\mathrm{a}} \\
154596^{\mathrm{a}} \\
923148^{\mathrm{a}} \\
1078629^{\mathrm{a}} \\
945495^{\mathrm{a}} \\
874736^{\mathrm{a}} \\
979168^{\mathrm{a}} \\
\end{array}$ & $\begin{array}{c}6232^{\mathrm{a}} \\
12776^{\mathrm{a}} \\
4560^{\mathrm{a}} \\
2610^{\mathrm{a}} \\
3447^{\mathrm{a}} \\
3270^{\mathrm{a}} \\
4471^{\mathrm{a}} \\
1788^{\mathrm{a}} \\
4867^{\mathrm{a}} \\
5835^{\mathrm{a}} \\
\end{array}$ & $\begin{array}{l}78827^{\mathrm{a}} \\
90662^{\mathrm{a}} \\
54750^{\mathrm{a}} \\
44251^{\mathrm{a}} \\
38595^{\mathrm{a}} \\
57034^{\mathrm{a}} \\
34130^{\mathrm{a}} \\
35513^{\mathrm{a}} \\
86260^{\mathrm{a}} \\
41873^{\mathrm{a}} \\
\end{array}$ \\
\hline $\begin{array}{c}\text { NPKS x C } \\
0 \text { Anna } \\
0 \text { Edna } \\
0 \text { Ohio } \\
25 \text { Anna } \\
25 \text { Edna } \\
25 \text { Ohio } \\
50 \text { Anna } \\
50 \text { Edna } \\
50 \text { Ohio } \\
75 \text { Anna } \\
75 \text { Edna } \\
75 \text { Ohio } \\
100 \text { Anna } \\
100 \text { Edna } \\
100 \text { Ohio }\end{array}$ & $\begin{array}{c}-58678^{\mathrm{a}} \\
- \\
103149^{\mathrm{a}} \\
56685^{\mathrm{a}} \\
66298^{\mathrm{a}} \\
99329^{\mathrm{a}} \\
64382^{\mathrm{a}} \\
64344^{\mathrm{a}} \\
88142^{\mathrm{a}} \\
53114^{\mathrm{a}} \\
57808^{\mathrm{a}} \\
74292^{\mathrm{a}} \\
58591^{\mathrm{a}} \\
50580^{\mathrm{bc}} \\
58197^{\mathrm{defg}}\end{array}$ & $\begin{array}{c}10562^{\mathrm{i}} \\
- \\
15402^{\mathrm{hi}} \\
23222^{\text {ggh }} \\
80818^{\mathrm{a}} \\
39259^{\mathrm{cd}} \\
25897^{\mathrm{efgh}} \\
69863^{\mathrm{a}} \\
36311^{\text {cde }} \\
19430^{\mathrm{ghi}} \\
53379^{\mathrm{b}} \\
34372^{\text {cdef }} \\
18719^{\text {ghi }} \\
44786^{\mathrm{d}} \\
28043^{\mathrm{d}}\end{array}$ & $\begin{array}{c}273789 \mathrm{e} \\
- \\
386071^{\mathrm{de}} \\
576210^{\mathrm{abc}} \\
432702^{\mathrm{cde}} \\
698772^{\mathrm{a}} \\
539804^{\mathrm{abcd}} \\
477731^{\mathrm{bcd}} \\
607951^{\mathrm{ab}} \\
479974^{\mathrm{bcd}} \\
474858^{\mathrm{bcd}} \\
538308^{\mathrm{abcd}} \\
404631^{\mathrm{de}} \\
431720^{\mathrm{cde}} \\
538308^{\mathrm{ab}}\end{array}$ & $\begin{array}{c}47384^{\mathrm{a}} \\
- \\
587494^{\mathrm{a}} \\
462357^{\mathrm{a}} \\
418615^{\mathrm{a}} \\
1512713^{\mathrm{a}} \\
380142^{\mathrm{a}} \\
371036^{\mathrm{a}} \\
1286054^{\mathrm{a}} \\
327347^{\mathrm{a}} \\
267365^{\mathrm{a}} \\
1195195^{\mathrm{a}} \\
246138^{\mathrm{a}} \\
147549^{\mathrm{a}} \\
1149680^{\mathrm{a}}\end{array}$ & $\begin{array}{c}394371^{\mathrm{a}} \\
- \\
280857^{\mathrm{a}} \\
531907^{\mathrm{a}} \\
951707^{\mathrm{a}} \\
639119^{\mathrm{a}} \\
680569^{\mathrm{a}} \\
1017338^{\mathrm{a}} \\
729410^{\mathrm{a}} \\
967246^{\mathrm{a}} \\
1196505^{\mathrm{a}} \\
815120^{\mathrm{a}} \\
889870^{\mathrm{a}} \\
1080244^{\mathrm{a}} \\
810743^{\mathrm{a}}\end{array}$ & $\begin{array}{c}3062^{\mathrm{a}} \\
- \\
12776^{\mathrm{a}} \\
13748^{\mathrm{a}} \\
11205^{\mathrm{a}} \\
17279^{\mathrm{a}} \\
10987^{\mathrm{a}} \\
11556^{\mathrm{a}} \\
16143^{\mathrm{a}} \\
10897^{\mathrm{a}} \\
9603^{\mathrm{a}} \\
15615^{\mathrm{a}} \\
7108^{\mathrm{a}} \\
6174^{\mathrm{a}} \\
14989^{\mathrm{a}}\end{array}$ & $\begin{array}{c}78827^{\mathrm{a}} \\
- \\
90662^{\mathrm{a}} \\
214035^{\mathrm{a}} \\
266270^{\mathrm{a}} \\
219817^{\mathrm{a}} \\
200745^{\mathrm{a}} \\
257992^{\mathrm{a}} \\
222622^{\mathrm{a}} \\
254232^{\mathrm{a}} \\
299310^{\mathrm{a}} \\
261001^{\mathrm{a}} \\
217627^{\mathrm{a}} \\
265747^{\mathrm{a}} \\
272456^{\mathrm{a}}\end{array}$ \\
\hline $\begin{array}{c}\text { F-value } \\
\text { NPKS } \\
B \\
\text { C } \\
\text { NPKS x B } \\
\text { NPKS x C } \\
\text { B x C } \\
\text { NPKS x B x } \\
\end{array}$ & $\begin{array}{l}0.05 \\
0.78 \\
0.01 \\
0.12 \\
0.46 \\
0.37 \\
0.46 \\
\end{array}$ & $\begin{array}{c}0.0001 \\
0.21 \\
0.0001 \\
0.32 \\
0.008 \\
0.37 \\
0.42 \\
\end{array}$ & $\begin{array}{c}0.01 \\
0.05 \\
0.0001 \\
0.05 \\
0.31 \\
0.87 \\
0.22 \\
\end{array}$ & $\begin{array}{c}0.01 \\
0.90 \\
0.0001 \\
0.31 \\
0.93 \\
0.33 \\
0.94 \\
\end{array}$ & $\begin{array}{c}0.0001 \\
1.00 \\
0.0001 \\
0.06 \\
0.91 \\
0.26 \\
0.90 \\
\end{array}$ & $\begin{array}{c}0.01 \\
0.75 \\
0.0001 \\
0.26 \\
0.72 \\
0.64 \\
0.85 \\
\end{array}$ & $\begin{array}{c}0.0001 \\
0.51 \\
0.05 \\
0.23 \\
0.91 \\
0.50 \\
0.24 \\
\end{array}$ \\
\hline
\end{tabular}

A) 5-(Pentafluoro-lambda 6 -sulfanyl)-2H-benzimidazole-2-thione, B) (2S,3R)-2,3-Dihydroxy-5oxohexanedioate, C) 2-(1-hydroxyethyl thiamine diphosphate (2-), D) Patuletin-3-glucosyl-(1-6) [apiosyl (1-2)]-glucoside, E) N-Acetyl-D-tryptophan, F) Spinacetin-3-glucosyl-(1-6) [apiosyl (1-2)]glucoside, G) Suvorexant 
Table $2 \boldsymbol{b}$. Concentration mean of compounds identified from three selected baby spinach cultivars with different levels of NPKS nutrition and Bacillus subtilis BD233 inoculation

\begin{tabular}{|c|c|c|c|c|c|c|c|}
\hline \multicolumn{8}{|c|}{ Parameters } \\
\hline & $\mathbf{H}$ & I & $\mathbf{J}$ & $\mathbf{K}$ & $\mathbf{L}$ & M & $\mathbf{N}$ \\
\hline $\operatorname{Mass}(\mathbf{m} / \mathbf{z})$ & 977 & 525 & 133 & 429 & 675 & 96 & 837 \\
\hline RT (min) & 6.40 & 6.84 & 0.86 & 7.40 & 7.40 & 0.70 & 7.02 \\
\hline Treatment & & & & & & & \\
\hline $\begin{array}{c}\text { NPKS } \\
0 \\
25 \\
50 \\
75 \\
100 \\
\end{array}$ & $\begin{array}{c}767784^{\mathrm{ab}} \\
857838^{\mathrm{a}} \\
702175^{\mathrm{b}} \\
643453^{\mathrm{bc}} \\
517751^{\mathrm{c}} \\
\end{array}$ & $\begin{array}{l}452420^{\mathrm{a}} \\
324722^{\mathrm{a}} \\
411397^{\mathrm{a}} \\
478901^{\mathrm{a}} \\
449850^{\mathrm{a}}\end{array}$ & $\begin{array}{l}5284^{\mathrm{a}} \\
4612^{\mathrm{ab}} \\
3813^{\mathrm{b}} \\
2797^{\mathrm{c}} \\
2877^{\mathrm{c}} \\
\end{array}$ & $\begin{array}{l}5214^{\mathrm{b}} \\
9008^{\mathrm{a}} \\
4632^{\mathrm{b}} \\
6010^{\mathrm{b}} \\
4488^{\mathrm{b}} \\
\end{array}$ & $\begin{array}{l}4965^{\mathrm{a}} \\
2415^{\mathrm{b}} \\
2239^{\mathrm{b}} \\
2136^{\mathrm{b}} \\
2076^{\mathrm{b}} \\
\end{array}$ & $\begin{array}{c}10374^{\mathrm{a}} \\
7370^{\mathrm{b}} \\
3057^{\mathrm{bc}} \\
1244^{\mathrm{cd}} \\
1456^{\mathrm{d}} \\
\end{array}$ & $\begin{array}{l}1896^{\mathrm{b}} \\
2978^{\mathrm{a}} \\
2080^{\mathrm{b}} \\
2058^{\mathrm{b}} \\
1727^{\mathrm{b}}\end{array}$ \\
\hline $\begin{array}{c}\text { Bacillus (B) } \\
B- \\
B+ \\
\end{array}$ & $\begin{array}{l}672596^{\mathrm{a}} \\
698819^{\mathrm{a}} \\
\end{array}$ & $\begin{array}{l}428948^{\mathrm{a}} \\
407322^{\mathrm{a}} \\
\end{array}$ & $\begin{array}{l}3615^{\mathrm{a}} \\
3642^{\mathrm{a}} \\
\end{array}$ & $\begin{array}{l}5223^{\mathrm{a}} \\
6780^{\mathrm{a}} \\
\end{array}$ & $\begin{array}{l}2445^{\mathrm{a}} \\
2309^{\mathrm{a}} \\
\end{array}$ & $\begin{array}{l}5934^{\mathrm{a}} \\
6227^{\mathrm{a}} \\
\end{array}$ & $\begin{array}{l}2198^{a} \\
2186^{a}\end{array}$ \\
\hline $\begin{array}{c}\text { Cultivar (C) } \\
\text { Anna } \\
\text { Edna } \\
\text { Ohio } \\
\end{array}$ & $\begin{array}{c}51961^{\mathrm{b}} \\
528361^{\mathrm{b}} \\
980733^{\mathrm{a}} \\
\end{array}$ & $\begin{array}{l}371454^{\mathrm{a}} \\
441268^{\mathrm{a}} \\
434503^{\mathrm{a}}\end{array}$ & $\begin{array}{l}3682^{a} \\
3184^{a} \\
4028^{a}\end{array}$ & $\begin{array}{l}2840^{\mathrm{b}} \\
6965^{\mathrm{a}} \\
7630^{\mathrm{a}} \\
\end{array}$ & $\begin{array}{l}2570^{\mathrm{a}} \\
2181^{\mathrm{a}} \\
2416^{\mathrm{a}} \\
\end{array}$ & $\begin{array}{l}7921^{\mathrm{a}} \\
5612^{\mathrm{b}} \\
5008^{\mathrm{b}} \\
\end{array}$ & $\begin{array}{l}1757^{\mathrm{b}} \\
2381^{\mathrm{a}} \\
2366^{\mathrm{a}}\end{array}$ \\
\hline $\begin{array}{c}\text { NPKS x } B \\
\text { 0B- } \\
\text { 0B+ } \\
25 \mathrm{~B}- \\
25 \mathrm{~B}+ \\
50 \mathrm{~B}- \\
50 \mathrm{~B}+ \\
75 \mathrm{~B}- \\
75 \mathrm{~B}+ \\
100 \mathrm{~B}- \\
100 \mathrm{~B}+ \\
\end{array}$ & $\begin{array}{c}640602^{\mathrm{a}} \\
1022148^{\mathrm{a}} \\
860826^{\mathrm{a}} \\
854851^{\mathrm{a}} \\
703846^{\mathrm{a}} \\
700503^{\mathrm{a}} \\
671590^{\mathrm{a}} \\
615316^{\mathrm{a}} \\
464786^{\mathrm{a}} \\
570716^{\mathrm{a}} \\
\end{array}$ & $\begin{array}{c}383143^{\mathrm{a}} \\
590975^{\mathrm{a}} \\
195521^{\mathrm{a}} \\
107535^{\mathrm{abc}} \\
230787^{\mathrm{a}} \\
144074^{\mathrm{a}} \\
245275^{\mathrm{a}} \\
127589^{\mathrm{ab}} \\
151048^{\mathrm{ab}} \\
82293^{\mathrm{a}}\end{array}$ & $\begin{array}{c}5591^{\mathrm{a}} \\
4671^{\mathrm{a}} \\
4467^{\mathrm{a}} \\
4757^{\mathrm{a}} \\
4019^{\mathrm{a}} \\
2747^{\mathrm{a}} \\
297368^{\mathrm{a}} \\
2847^{\mathrm{a}} \\
2570^{\mathrm{a}} \\
3183^{\mathrm{a}} \\
\end{array}$ & $\begin{array}{c}2774^{\mathrm{a}} \\
10092^{\mathrm{a}} \\
8921^{\mathrm{a}} \\
9095^{\mathrm{a}} \\
3244^{\mathrm{a}} \\
5040^{\mathrm{a}} \\
671590^{\mathrm{a}} \\
6981^{\mathrm{a}} \\
4502^{\mathrm{a}} \\
4475^{\mathrm{a}} \\
\end{array}$ & $\begin{array}{c}5271^{\mathrm{a}} \\
4352^{\mathrm{a}} \\
2429^{\mathrm{a}} \\
2401^{\mathrm{a}} \\
2248^{\mathrm{a}} \\
2034^{\mathrm{a}} \\
441871^{\mathrm{a}} \\
2237^{\mathrm{a}} \\
2127^{\mathrm{a}} \\
2025^{\mathrm{a}} \\
\end{array}$ & $\begin{array}{c}10369^{\mathrm{a}} \\
10383^{\mathrm{a}} \\
5720^{\mathrm{a}} \\
9020^{\mathrm{a}} \\
7109^{\mathrm{a}} \\
6476^{\mathrm{a}} \\
5285^{\mathrm{a}} \\
4232^{\mathrm{a}} \\
4146^{\mathrm{a}} \\
4489^{\mathrm{a}} \\
\end{array}$ & $\begin{array}{c}1710^{\text {ef }} \\
2267^{\text {cd }} \\
1533^{\mathrm{a}} \\
1920^{\text {ab }} \\
2170^{\text {cde }} \\
1990^{\text {cdef }} \\
2393^{\text {bc }} \\
1724^{\text {def }} \\
1533^{\mathrm{f}} \\
1920^{\text {cdef }}\end{array}$ \\
\hline $\begin{array}{c}\text { NPKS x C } \\
0 \text { Anna } \\
0 \text { Edna } \\
0 \text { Ohio } \\
25 \text { Anna } \\
25 \text { Edna } \\
25 \text { Ohio } \\
50 \text { Anna } \\
50 \text { Edna } \\
50 \text { Ohio } \\
75 \text { Anna } \\
75 \text { Edna } \\
75 \text { Ohio } \\
100 \text { Anna } \\
100 \text { Edna } \\
100 \text { Ohio }\end{array}$ & $\begin{array}{c}640602^{\mathrm{de}} \\
- \\
1022148^{\mathrm{ab}} \\
728881^{\mathrm{cd}} \\
635646^{\mathrm{de}} \\
1131614^{\mathrm{a}} \\
542803^{\mathrm{def}} \\
575829^{\mathrm{de}} \\
987892^{\mathrm{ab}} \\
485793^{\mathrm{ef}} \\
526041^{\mathrm{def}} \\
908462^{\mathrm{bc}} \\
356676^{\mathrm{f}} \\
349687^{\mathrm{f}} \\
846890^{\mathrm{bc}}\end{array}$ & $\begin{array}{c}383143^{\mathrm{a}} \\
- \\
590975^{\mathrm{a}} \\
126845^{\mathrm{a}} \\
260222^{\mathrm{a}} \\
468372^{\mathrm{a}} \\
350176^{\mathrm{a}} \\
469323^{\mathrm{a}} \\
414691^{\mathrm{a}} \\
489069^{\mathrm{a}} \\
489069^{\mathrm{a}} \\
354418^{\mathrm{a}} \\
420981^{\mathrm{a}} \\
475623^{\mathrm{a}} \\
452945^{\mathrm{a}}\end{array}$ & $\begin{array}{c}5591^{\mathrm{a}} \\
- \\
4671^{\mathrm{a}} \\
6152^{\mathrm{a}} \\
3457^{\mathrm{a}} \\
5150^{\mathrm{a}} \\
3285^{\mathrm{a}} \\
3319^{\mathrm{a}} \\
4836^{\mathrm{a}} \\
2605^{\mathrm{a}} \\
3056^{\mathrm{a}} \\
2617^{\mathrm{a}} \\
2696^{\mathrm{a}} \\
2866^{\mathrm{a}} \\
3069^{\mathrm{a}}\end{array}$ & $\begin{array}{c}2774^{\mathrm{a}} \\
- \\
10092^{\mathrm{a}} \\
7628^{\mathrm{a}} \\
6683 \mathrm{a} \\
11884^{\mathrm{a}} \\
614^{\mathrm{a}} \\
7154^{\mathrm{a}} \\
6127^{\mathrm{a}} \\
3381^{\mathrm{a}} \\
8330^{\mathrm{a}} \\
5084^{\mathrm{a}} \\
2298^{\mathrm{a}} \\
5423^{\mathrm{a}} \\
5744^{\mathrm{a}}\end{array}$ & $\begin{array}{c}5271^{\mathrm{a}} \\
- \\
4352^{\mathrm{a}} \\
2299^{\mathrm{a}} \\
2352^{\mathrm{a}} \\
2524^{\mathrm{a}} \\
2245^{\mathrm{a}} \\
2124^{\mathrm{a}} \\
2349^{\mathrm{a}} \\
2051^{\mathrm{a}} \\
2065^{\mathrm{a}} \\
2287^{\mathrm{a}} \\
2069^{\mathrm{a}} \\
2167^{\mathrm{a}} \\
1992^{\mathrm{a}}\end{array}$ & $\begin{array}{c}10369^{\mathrm{a}} \\
- \\
10383^{\mathrm{a}} \\
12150^{\mathrm{a}} \\
8245^{\mathrm{a}} \\
4583^{\mathrm{a}} \\
9910^{\mathrm{a}} \\
4968^{\mathrm{a}} \\
5500^{\mathrm{a}} \\
4829^{\mathrm{a}} \\
4982^{\mathrm{a}} \\
4426^{\mathrm{a}} \\
4914^{\mathrm{a}} \\
3750^{\mathrm{a}} \\
4288^{\mathrm{a}}\end{array}$ & $\begin{array}{c}1710 \mathrm{~d}^{\text {efg }} \\
- \\
226^{\mathrm{bcd}} \\
2955^{\mathrm{a}} \\
2839^{\mathrm{a}} \\
3126^{\mathrm{a}} \\
1575^{\mathrm{fg}} \\
2609^{\mathrm{abc}} \\
2056^{\mathrm{cdef}} \\
1402^{\mathrm{g}} \\
2551^{\mathrm{abc}} \\
1935^{\mathrm{defg}} \\
1629^{\text {efg }} \\
1369^{\mathrm{g}} \\
2182^{\text {cde }}\end{array}$ \\
\hline $\begin{array}{c}\text { F-value } \\
\text { NPKS } \\
B \\
C \\
\text { NPKS x B } \\
\text { NPKS x C } \\
\text { B x C } \\
\text { NPKS x B x }\end{array}$ & $\begin{array}{c}0.0001 \\
0.68 \\
0.02 \\
0.43 \\
0.00 \\
0.36 \\
0.87 \\
\end{array}$ & $\begin{array}{l}0.20 \\
0.65 \\
0.43 \\
0.25 \\
0.16 \\
0.44 \\
0.34 \\
\end{array}$ & $\begin{array}{c}0.001 \\
0.90 \\
0.93 \\
0.95 \\
1.00 \\
0.91 \\
1.01 \\
\end{array}$ & $\begin{array}{c}0.0001 \\
0.47 \\
0.01 \\
0.18 \\
0.09 \\
0.98 \\
0.78 \\
\end{array}$ & $\begin{array}{l}0.05 \\
0.90 \\
0.93 \\
0.95 \\
1.01 \\
0.91 \\
1.00 \\
\end{array}$ & $\begin{array}{c}0.0001 \\
0.47 \\
0.01 \\
0.18 \\
0.09 \\
0.98 \\
0.79 \\
\end{array}$ & $\begin{array}{c}0.0001 \\
0.82 \\
0.002 \\
0.01 \\
0.05 \\
0.80 \\
0.57 \\
\end{array}$ \\
\hline
\end{tabular}

H) Spinacetin-3-(2фф-feroylglucosyl) (1-6) [apiosyl(1-2)]-glucoside, I) 4-(beta-D-Glucopyranosyloxy)2-hydroxy-6-pentadecylbenzoic acid, J) S)-Malate, K) 4-[3-(Benzyloxy)-1-(beta-D-glucopyranosyloxy) prosody] butanoic acid, L) Diethyl (\{4-(6-oxo-7,11-diazatricyclo [7.3.1.0 2,7 ] trideca-2,4-dien-11-yl)3-[(3,4,5-trimethoxybenzoyl) amino] benzoyl $\}$ amino) malonate, M) 3- $\$ 1^{\wedge}\{1\}$-oxidanyl-4,5didehydroisothiazole, N) 5-[(2-\{[2-Acetamido-2-deoxy-3-O-(beta-D-galactopyranosyl)-alpha-Dgalactopyranosyl] oxy ethyl) carbamoyl]-2-[6-(dimethylamino)-3-(dimethyliminio)-3H-xanthen-9-yl] benzoa 
$\mathrm{N}$-Acetyl-D-tryptophan (m/z 245, Rt. 5.55) was reported by Okazaki et al. (2009) on spinach leaves when treated by altering the ratio of $\mathrm{NH}_{4}{ }^{+} / \mathrm{NO}_{3}{ }^{-}$in the culture solution. However, 5-[(2-\{[2-Acetamido-2-deoxy-3-O-(beta-D-galactopyranosyl)-alpha-Dgalactopyranosyl] oxy ethyl) carbamoyl]-2-[6-(dimethylamino)-3-(dimethyliminio)$3 \mathrm{H}$-xanthen-9-yl] benzoate (m/z 837, Rt. 7.02), 5-(Pentafluoro-lambda 6 -sulfanyl)-2Hbenzimidazole-2-thione (m/z 272, Rt. 0.61), (2S,3R)-2,3-Dihydroxy-5-oxohexanedioate (m/z 191, Rt. 1.06), 2-(1-hydroxyethyl)thiamine diphosphate(2-) (m/z 465, Rt. 5.20), suvorexant (m/z 449, Rt. 6.20), 4-(beta-D-Glucopyranosyloxy)-2-hydroxy-6pentadecylbenzoic acid (m/z 525, Rt. 6.84), 1-(5"-Phosphoribosyl)-5-amino-4imidazolecarboxamide (m/z 337, Rt. 5.84), Diacetylacteoside (m/z 707, Rt. 6.98),5,7Dihydroxy-2-(4-hydroxy-3,5-dimethoxyphenyl)-4-oxo-4H-chromen-3-yl-beta-D-

erythro-hexopyranosiduronic acid (m/z 521, Rt. 7.09), 2-(3,4-Dihydroxyphenyl)-5,7dihydroxy-6-methoxy-4-oxo-4H-chromen-3-yl

6-O-acetyl-beta-D-erythrohexopyranoside (m/z 535, Rt. 7.34), Narirutin (m/z 579, Rt. 5.60), 4-[3-(Benzyloxy)-1(beta-D-glucopyranosyloxy)propoxy]butanoic acid (m/z 429, Rt. 7.40), 4[(Nitrooxy)methyl]benzyl N-\{(5Z)-7-[(1R,2R,3R,5S)-3,5-dihydroxy-2-\{(1E,3R)-3hydroxy-4-[3-(trifluoromethyl)phenoxy]-1-buten-1-yl \}cyclopentyl]-5-

heptenoyl \}glycinate (m/z 679, Rt. 6.71), were not identified in previous studies on baby spinach.

PLS-DA score plot was used to assess the significance of class discrimination (Fig. 1A,B). The supervised comparison of baby spinach treated with different levels of fertilizers and Bacillus subtilis BD233 revealed distinct grouping among the control, $33 \mathrm{~N}: 33 \mathrm{P}: 45 \mathrm{~K}: 7 \mathrm{~S} \mathrm{~kg} / \mathrm{ha}$ and $45 \mathrm{~N}: 45 \mathrm{P}: 60 \mathrm{~K}: 10 \mathrm{~S} \mathrm{~kg} / \mathrm{ha}$. The first component of the data was effective in separating the control from samples treated with fertilizers. Most of the fertilizer treated samples are on the left side of the plot and samples treated with fertilizers and B. subtilis on the right side of the plot (Fig. 1A,B).

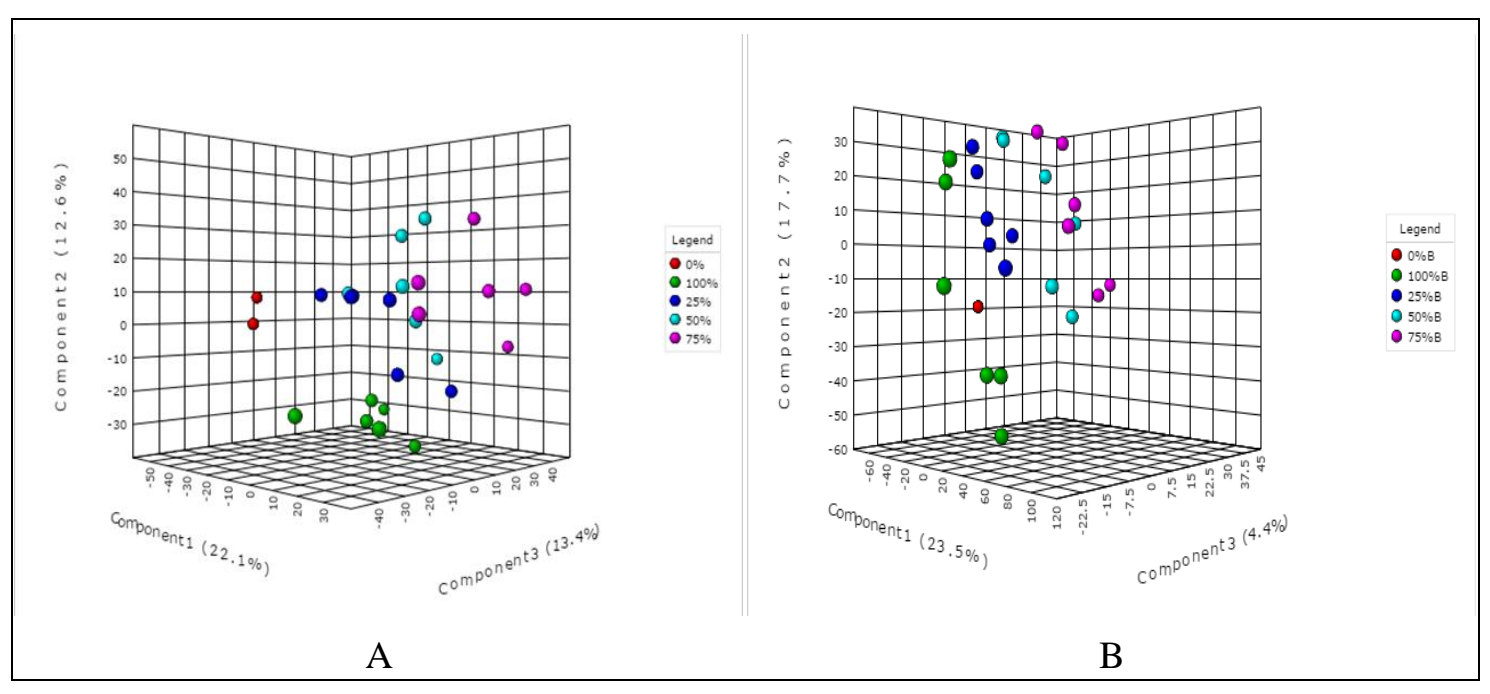

Figure 1. Partial Least Squares - Discriminant Analysis (PLS-DA) 3D scores plot from LC-MS for baby spinach treated with different rates of fertilizers and Bacillus subtilis BD233.

$0 \%=0 \mathrm{~N}: 0 P: 0 \mathrm{~K}: 0 \mathrm{~S} \mathrm{~kg} / \mathrm{ha}, 25 \%=11 \mathrm{~N}: 11 \mathrm{P}: 15 \mathrm{~K}: 2 \mathrm{~S} \mathrm{~kg} / \mathrm{ha}, 50 \%=22 \mathrm{~N}: 22 \mathrm{P}: 30 \mathrm{~K}: 5 \mathrm{~S} \mathrm{~kg} / \mathrm{ha}, 75 \%$ $=33 \mathrm{~N}: 33 \mathrm{P}: 45 \mathrm{~K}: 7 \mathrm{~S} \mathrm{~kg} / \mathrm{ha}, 100 \%=45 \mathrm{~N}: 45 P: 60 \mathrm{~K}: 10 \mathrm{~S} \mathrm{~kg} / \mathrm{ha}$ and $\mathrm{B}=$ Bacillus subtilis BD233 


\section{Conclusion}

The current study showed evidence of differences in the chlorophyll content of the baby spinach cv. Anna, Edna and Ohio with the same treatments, however, no differences observed in stomatal conductance and total biomass among the three cultivars. Inoculation with $B$. subtilis also yielded the same results where no significant differences were observed in chlorophyll content, stomatal conductance and total biomass. Fertilizer application at $33 \mathrm{~N}: 33 \mathrm{P}: 45 \mathrm{~K}: 7 \mathrm{~S} \mathrm{~kg} / \mathrm{ha}$ and $45 \mathrm{~N}: 45 \mathrm{P}: 60 \mathrm{~K}: 10 \mathrm{~S} \mathrm{~kg} / \mathrm{ha}$ influenced the physiological parameters when compared to the control of the study. The results confirmed that plants treated with $11 \mathrm{~N}: 11 \mathrm{P}: 15 \mathrm{~K}: 2 \mathrm{~S} \mathrm{~kg} / \mathrm{ha}$ and $22 \mathrm{~N}: 22 \mathrm{P}: 30 \mathrm{~K}: 5 \mathrm{~S} \mathrm{~kg} / \mathrm{ha}$ baby spinach cv. Ohio is rich in flavonoids such as patuletin-3-glucosyl-(1-6)[apiosyl(12)]-glucoside (m/z 787, Rt. 5.39), spinacetin-3-glucosyl-(1-6)[apiosyl(1-2)]-glucoside $(\mathrm{m} / \mathrm{z}$ 801, Rt. 5.94) and spinacetin-3-(2фф-feroylglucosyl)(1-6)[apiosyl(1-2)]-glucoside $(\mathrm{m} / \mathrm{z}$ 977, Rt. 6.40), which have the potential protective response against cancer and heart diseases and also their antioxidative properties. Meanwhile, baby spinach cv. Anna, Edna and Ohio were found to be highly concentrated with amino acids such as $(\mathrm{S})$-Malate $(\mathrm{m} / \mathrm{z}$ 133, Rt. 0.86) when no treatment applied, 2-(1-hydroxyethyl)thiamine diphosphate(2-) (m/z 465, Rt. 5.20) in all fertilizer application levels except the control, N-Acetyl-Dtryptophan (m/z 245, Rt. 5.55) when treated with $11 \mathrm{~N}: 11 \mathrm{P}: 15 \mathrm{~K}: 2 \mathrm{~S} \mathrm{~kg} / \mathrm{ha}$ and Diethyl (\{4(6-oxo-7,11-diazatricyclo[7.3.1.0 2,7 ]trideca-2,4-dien-11-yl)-3-[(3,4,5

trimethoxybenzoyl) amino] benzoyl \} amino) malonate (m/z 675, Rt. 7.40) when no treatment applied, which are essential for tissue growth and repair, as well to assist in muscle building. There was a significant difference in the compounds among cultivars and NPKS fertilization. The study recommends that fertilizer at $22 \mathrm{~N}: 22 \mathrm{P}: 30 \mathrm{~K}: 5 \mathrm{~S} \mathrm{~kg} / \mathrm{ha}$ and $33 \mathrm{~N}: 33 \mathrm{P}: 45 \mathrm{~K}: 7 \mathrm{~S} \mathrm{~kg} / \mathrm{ha}$ amended with $B$. subtilis inoculation be considered when cultivating baby spinach. However, the metabolite profiling of baby spinach needs further investigation to determine the influence of $B$. subtilis application and levels of NPKS on flavor characterization.

Acknowledgements. The authors would like to thank SAKATA for providing seeds for the experiment and NRF (Grant No: 100503) for providing funds for this study.

Conflict of interests. The authors declared that there is no conflict of interests regarding the publication of this paper.

\section{REFERENCES}

[1] Anjum, M. A., Sajjad, M. R., Akhtar, N., Qureshi, M. A., Iqbal, A., Jami, A. R. (2007): Response of cotton to plant growth promoting rhizobacteria (PGPR) under different levels of nitrogen. - Journal of Agricultural Research 45: 135-143.

[2] Bashan, Y., Huang, P., Kloepper, J. W., de-Bashan, L. (2017): A proposal for avoiding fresh-weight measurements when reporting the effect of plant growth-promoting (rhizo) bacteria on growth promotion of plants. - Biology and Fertility of Soils 53: 1-2.

[3] Bergquist, S. A. M., Gertsson, U. E., Knuthsen, P., Olsson, M. E. (2005): Flavonoids in Baby spinach (Spinacia oleracea L.): Changes during Plant Growth and Storage. - Journal of Agricultural and Food Chemistry 53: 9459-9464.

[4] Bergquist, S. (2006): Bioactive compounds in baby spinach (Spinacia oleracea L.) effects of pre- and postharvest factors. - Journal of the Science of Food and Agriculture 86: 346355 . 
[5] Boroujerdnia, M., Ansari, N. A. (2007): Effect of different levels of nitrogen fertilizer and cultivars on growth, yield and yield components of romaine lettuce (Lactuca sativa L.). Middle Eastern and Russian Journal of Plant Science and Biotechnology 1(2): 47-53.

[6] Çakmakçi, R., Erat, M., Erdogan, Ü., Dönmez, M. F. (2007): The influence of plant growthpromoting rhizobacteria on growth and enzyme activities in wheat and spinach plants. Journal of Plant Nutrition and Soil Science 170: 288-295.

[7] Canbolat, M. Y., Bilen, S., Çakmakçı, R., Şahin, F., Aydın, A. (2006): Effect of plant growth-promoting bacteria and soil compaction on barley seedling growth, nutrient uptake, soil properties and rhizosphere microflora. - Biology and Fertility of Soils 42: 350-357.

[8] Diacono, M., Montemurro, F. (2010): Long-term effects of organic amendments on soil fertility. A review. - Agronomy for Sustainable Development 30: 401-422.

[9] Ekinci, M., Turan, M., Yildirim, E., Güneú, A., Kotan, R., Dursun, A. (2014): Effect of plant growth promoting rhizobacteria on growth, nutrient, organic acid, amino acid and hormone content of cauliflower (Brassica oleracea L. var. Botrytis) transplants. - Acta Sci Pol Hortorum Cultus 13(6): 71-85.

[10] Gairola, S., Umar, S., Suryapani, S. (2009): Nitrate accumulation, growth and leaf quality of spinach beet (Beta vulgaris Linn.) as affected by NPK fertilization with special reference to potassium. - Indian Journal of Science and Technology 2: 35-40.

[11] García-López, A. M., Delgado, A. (2016): Effect of Bacillus subtilis on phosphorus uptake by cucumber as affected by iron oxides and the solubility of the phosphorus source. Agricultural and Food Science 25: 216-224.

[12] Gloria, P. M., Ibrahim, I. I., Zwalnan, D. N., Jameela, A., Henry, U. I. (2017): Effect of different levels of NPK fertilizer on the growth and yield of okra (Abelmoschus esculentus L.). - Int J Adv Acad Res. Sciences, Technology \& Engineering 3(1): 1-8.

[13] Kalt, W. (2005): Effects of production and processing factors on major fruit and vegetable antioxidants. - Journal of food science 70: 11-19.

[14] Kerr, B. (2014): What is Spinach? - Farmer's Weekly, March 2014 Issue, South Africa.

[15] Khan, N. A., Nazar, R., Anjum, N. A. (2009): Growth, photosynthesis and antioxidant metabolism in mustard (Brassica juncea L.) cultivars differing in ATP-sulfurylase activity under salinity stress. - Scientia Horticulturae 122: 455-460.

[16] King, S., Davis, A., Perkins-Velazie, P., Helms, A., Stein, L. (2008): Effects of mineral nutrition on carotenoid content in spinach. - HortScience 43: 1246.

[17] Makus, D. J. (2013): Spinach leaf quality and yield is improved by supplemental gypsum application in two soil type in semi-arid South Texas. - Subtropical Plant Science 65: 2430 .

[18] Masufi, N. M., Mudau, A. R., Araya, H. T., Mudau, F. N. (2019): The developmental growth and quality assessment of five selected cultivars of baby spinach grown in the north of Pretoria, Gauteng Province, South Africa. - South African Journal of Plant and Soil (In press).

[19] Mncwangi, N. P., Viljoen, A. M., Zhao, J., Vermaak, I., Chen, W., Khan, I. (2014): What the devil is in your phytomedicine? Exploring species substitution in Harpagophytum through chemometric modeling of 1H-NMR and UHPLC-MS datasets. - Phytochemistry 106: 104-115.

[20] Mudau, A. R., Nkomo, M., Soundy, P., Araya, H. T., Ngezimana, W., Mudau, F. N. (2015): Influence of post-harvest storage temperature and duration on quality of baby spinach. HortTechnology 25: 665-670.

[21] Nemadodzi, L. E., Araya, H., Nkomo, M., Ngezima, W., Mudau, F. N. (2017): Nitrogen, phosphorus, and potassium effects on the physiology and biomass yield of baby spinach (Spinacia oleracea L.). - Journal of Plant Nutrition 40(14): 2033-2044.

[22] Okazaki, K., Oka, N., Shinano, T., Osaki, M., Takebe, M. (2009): Metabolite profiling of spinach (Spinacea oleracea L.) leaves by altering the ration of NH4+/NO3- in the culture solution. - Soil Science and Plant Nutrition 55: 496-504. 
[23] Porcel, R., Zamarreño, A. M., García-Mina, J. M., Aroca, R. (2014): Involvement of plant endogenous ABA in Bacillus megaterium PGPR activity in tomato plants. - BMC Plant Biology 14: 36.

[24] Pramanik, K., Bera, A. K. (2013): Effect of seedling age and nitrogen fertilizer on growth, chlorophyll content, yield and economics of hybrid rice (Oryza sativa L.). - Journal of Agronomy and Plant Production 4(5): 3489-3499.

[25] Pupathy, U. T., Radziah, O. (2015): Growth response of corn to nitrogen-fixing bacteria enriched compost. - Asian Journal of Crop Science 7: 72-80.

[26] Schrader, W., Mayberry, K. S. (2003): Beet and Swiss chard production in California. University of California, ANR Publication 8096.

[27] Sedibe, M. M. (2012): Yield and quality response of hydroponically grown rose geranium (Pelargonium Sp.) to changes in the nutrient solution and shading. - Doctoral Thesis (Soil, Crop and Climate Sciences), University of the Free State.

[28] Sedibe, M. M., Allemann, J. (2012): Yield and quality response of rose geranium (Pelargonium Sp.) to sulphur and phosphorus application. - South African Journal of Plant and Soil 29: 151-156.

[29] Shine, M. B., Guruprasad, K. N. (2012): Impact of pre-sowing magnetic field exposure of seeds to stationary magnetic field on growth, reactive oxygen species and photosynthesis of maize under field conditions. - Acta Physiologiae Plantarum 34: 255-265.

[30] Singh, K., Singh, P. P., Beg, S. U., Kumar, D., Patra, D. D. (2004): Effect of NPK fertilizers on growth, oil yield and quality of French basil (Ocimum basilicum L.). - Journal of Spices Aromatic Crops 13(1): 52-54.

[31] Singh, D. B., Ahmed, N., Singh, S. R., Mir, K. A., Lal, S. (2014): Variation in chlorophyll and carotenoid contents in kale (Brassica oleracea) as influenced by cultivars and harvesting dates. - Indian Journal of Agricultural Sciences 84(10): 1178-81.

[32] Turan, M., Ekinci, M., Yildirim, E., Güneş, A., Karagöz, K., Kotan, R., Dursun, A. (2014): Plant growth-promoting rhizobacteria improved growth, nutrient, and hormone content of cabbage (Brassica oleracea) seedlings. - Turkish Journal of Agriculture and Forestry 38: 327-333.

[33] Wu, Y. N., Feng, Y. L., Paré, P. W., Chen, Y. L., Xu, R., Wu, S., Wang, S. M., Zhao, Q., Li, H. R., Wang, Y. Q., Zhang, J. L. (2016): Beneficial soil microbe promotes seed germination, plant growth and photosynthesis in herbal crop Codonopsis pilosula. - Crop and Pasture Science 67(1): 91-98.

[34] Yakhin, O. I., Lubyanov, A. A., Yakhin, I. A. (2016): Biostimulants in agrotechnologies: problems, solutions, outlook. - Agrochemical Her 1: 15-21. Available online at: http://www.agrochemv.ru/en/nomer/2016/1; http://elibrary.ru/item.asp?id=25940647 (Accessed 16 March 2019).

[35] Zhang, H., Xie, X., Kim, M. S., Kornyeyev, D. A., Holaday, S., Paré, P. W. (2008): Soil bacteria augment Arabidopsis photosynthesis by decreasing glucose sensing and abscisic acid levels in plants. - The Plant Journal 56: 264-273.

[36] Zikalala, B. O., Nkomo, M., Araya, H., Ngezimana, W., Mudau, F. N. (2016): Nutritional quality of baby spinach (Spinacia oleracea L.) as affected by nitrogen, phosphorus and potassium fertilisation. - South African Journal of Plant and Soil 34(2): 79-86. 